\title{
Numeral System Change in Arithmetic and Matricial Formalism
}

\author{
Raoelina Andriambololona, Ravo Tokiniaina Ranaivoson, Hanitriarivo Rakotoson
}

Theoretical Physics Department, Institut National des Sciences et Techniques Nucléaires (INSTN-Madagascar), Antananarivo, Madagascar

\section{Email address:}

instn@moov.mg (Raoelina Andriambololona), raoelinasp@yahoo.fr (Raoelina Andriambololona), jacquelineraoelina@hotmail.com (Raoelina Andriambololona), tokhiniaina@gmail.com (R. T. Ranaivoson), infotsara@gmail.com (H. Rakotoson)

\section{To cite this article:}

Raoelina Andriambololona, Ravo Tokiniaina Ranaivoson, Hanitriarivo Rakotoson. Numeral System Change in Arithmetic and Matricial Formalism. Pure and Applied Mathematics Journal. Vol. 5, No. 3, 2016, pp. 87-92. doi: 10.11648/j.pamj.20160503.15

Received: May 12, 2016; Accepted: May 25, 2016; Published: June 7, 2016

Abstract: The main goal of this paper is to present a method to tackle the numeral system change problem using matricial formalism. In a previous work, we have described an approach which permits to use matricial formalism and matricial calculation in writing numeration and arithmetic. The present paper is focused on the study of the problem of numeral system change in the framework of this approach. The cases of integer numbers and of more general numbers are given.

Keywords: Numeral System, Arithmetic, Radix, Matricial Formalism, Basis Change

\section{Introduction}

In a previous paper [1] we have presented an approach which permits the introduction of some tools of linear algebra like matricial formalism and matricial calculation [2], [3], [4] in the problem of writing numeration and arithmetics [5], [6], [7], [8]. This approach is based on the work performed by Raoelina Andriambololona since 1986 [9] [10], [11]. In the present work, our goal is to study the problem of numeral system change in the framework of this approach.

It was shown in our work [1] that, considering a numeral system with the radix $b$, a number $a$ can be considered as the product of a row matrix $W_{b}(a)$ and a column matrix $B$ built with the successive powers of $b$

$$
a=\sum_{s=s_{1}}^{s_{2}} a_{s} b^{s}=W_{b}(a) B=\left[\begin{array}{lll}
a_{s_{2}} & \ldots & a_{s_{1}}
\end{array}\right]\left[\begin{array}{c}
b^{s_{2}} \\
b^{s_{2}-1} \\
\cdot \\
\vdots \\
b^{s_{1}+1} \\
b^{s_{1}}
\end{array}\right]
$$

The row matrix $W_{b}(a)$ is the writing or the representation of the intrinsic number $a$ in the numeral system with radix $b$.

Another numeral system corresponding to another radix $b^{\prime}$ can be considered. Then in this new numeral system, the number $a$ will be represented by another row matrix $W_{b^{\prime}}(a)$.

$$
a=\sum_{s=s_{1}^{\prime}}^{s_{2}^{\prime}} a_{s}^{\prime} b^{\prime s}=W_{b^{\prime}}(a) B^{\prime}=\left[\begin{array}{lll}
a_{s_{2}^{\prime}}^{\prime} & \ldots & a_{s_{1}^{\prime}}^{\prime}
\end{array}\right]\left[\begin{array}{c}
b^{\prime s_{2}^{\prime}} \\
b^{\prime s_{2}^{\prime}-1} \\
\vdots \\
\vdots \\
b^{\prime s_{1}^{\prime}+1} \\
b^{\prime s_{1}^{\prime}}
\end{array}\right]
$$

In general $W_{b^{\prime}}(a) \neq W_{b}(a)$ and $B \neq B^{\prime}$ when $b \neq b^{\prime}$. The problem of numeral system change, that we tackle in the present paper, is to establish the relationship between $B$ and $B^{\prime}$ on one hand and $W_{b}(a)$ and $W_{b^{\prime}}(a)$ on another hand.

\section{System Change: Case of Integers}

Let us consider two systems of numeration corresponding respectively to two different radices $b$ and $b^{\prime}$. Let $a$ be an integer number. Let us denote respectively $W_{b}(a)$ and $W_{b^{\prime}}(a)$ the row matrices representing the number $a$ respectively in each of those numeral systems and let $B$ and $B^{\prime}$ be the column matrices representing respectively the powers of $b$ and $b^{\prime}$

$$
\begin{aligned}
& W_{b}(a)=\left[\begin{array}{lllll}
a_{n} & a_{n-1} & \ldots \ldots & a_{1} & a_{0}
\end{array}\right] \\
& W_{b^{\prime}}(a)=\left[\begin{array}{lllll}
a_{n^{\prime}}^{\prime} & a_{n^{\prime}-1}^{\prime} & \ldots \ldots & a_{1}^{\prime} & a_{0}^{\prime}
\end{array}\right]
\end{aligned}
$$




$$
\begin{gathered}
B=\left[\begin{array}{c}
b^{n} \\
b^{n-1} \\
\cdot \\
\vdots \\
b \\
b^{0}
\end{array}\right] \\
B^{\prime}=\left[\begin{array}{c}
b^{n^{\prime}} \\
b^{\prime n^{\prime}-1} \\
\cdot \\
\vdots \\
b^{\prime 1} \\
b^{\prime 0}
\end{array}\right] \\
a=W_{b}(a) B=W_{b^{\prime}}(a) B^{\prime}
\end{gathered}
$$

As previously mentioned in the introduction, our goal is to find the relationship between $B$ and $B^{\prime}$ on one hand and $W_{b}(a)$ and $W_{b^{\prime}}(a)$ on another hand.

Let us consider the relations (3), (4), (5) and (6). The numbers $n$ and $n^{\prime}$ are not necessary equal. The choice of $n$ and $n^{\prime}$ is fixed by the basis change matrix $[P]$ defined by the relation

$$
B^{\prime}=[P] B
$$

Generally, the matrix $[P]$ has an indefinite dimension. However, as it will be shown below, a rectangular matrix can be chosen.

The expressions of the number $a$ is

$$
a=W_{b}(a) B=W_{b^{\prime}}(a) B^{\prime}=W_{b^{\prime}}(a)[P] B
$$

As $B$ and $B^{\prime}$ are basis, it can be deduced that

$$
W_{b}(a)=W_{b^{\prime}}(a)[P]
$$

The writing $W_{b}(a)$ of $a$ in the numeral system with the radix $b$ is obtained immediately from the numeral system with the radix $b^{\prime}$ using matricial calculation.

Many elements of the matrix $[P]$ are null but in practical calculation, this matrix can be reduced to a rectangular matrix $n^{\prime} \times n$ with elements $P_{j}^{i}$. The numbers $n^{\prime}$ and $n$ are chosen such as we take into account all of non-null contributions in the calculations of $W_{b}(a)$ and $W_{b^{\prime}}(a)$.

$W_{b}(a)$ and $W_{b^{\prime}}(a)$ are row matrices respectively with $n$ and $n^{\prime}$ columns; we complete with zeros if necessary.

There are two cases.

$1^{\text {st }}$ case: the term

$$
a_{j}=\sum_{k=1}^{n^{\prime}} a_{k}^{\prime} P_{j}^{k}
$$

is less than $b . a_{j}$ is obtained immediately without performing any calculation.

$2^{\text {nd }}$ case: the term

$$
a_{j}=\sum_{k=1}^{n^{\prime}} a_{k}^{\prime} P_{j}^{k}
$$

is such as $b \leq a_{j}<b^{2}$

Then

$$
a_{j}=q_{j} b+r_{j}
$$

with

$$
0<q_{j} \leq b-1 \quad 0 \leq r_{j}<b-1
$$

in the numeral system with the radix $b$, we have

$$
a_{j} b^{j}=\left(q_{j} b+r_{j}\right) b^{j}=q_{j} b^{j+1}+r_{j} b^{j}
$$

The remainder $q_{j}$ is to be brought into the $(j+2)^{t h}$ column from the right handside and $r_{j}$ is the figure to be put at the $(j+1)^{\text {th }}$ column from the right handside.

Generalization: let us consider

$$
a_{j}=q_{j+l} b^{l}+q_{j+l-1} b^{l-1}+\ldots+q_{1} b^{1}+q_{0} b^{0}
$$

where

$$
q_{k} \leq b-1 k=\{j+l, \ldots, 0\}
$$

then

$$
a_{j} b^{j}=q_{j+l} b^{j+l}+q_{j+l-1} b^{j+l}+q_{1} b^{j+1}+q_{0} b^{j}
$$

It means that

-the number at the $(j+1)^{t h}$ column from the right handside is $q_{0}$

-the number at the $(j+2)^{\text {th }}$ column from the right handside is $q_{1}$

-the number at the $(j+1+l)^{t h}$ column from the right handside is $q_{l}$

The $q_{j+l}, q_{j+l-1}, \ldots, q_{1}$ are the "remainders" which must be added to the other contributions. The calculation rules which must be utilized can be seen immediately. We are led to perform additions and multiplications in respecting the places of the figures and to add the necessary remainders (it is the current rules for addition in arithmetic). The simplicity of the method will be illustrated by some examples. The calculations may be performed with a microcomputer.

Remarks: There is one and only one matrix $\left[P^{\prime}\right]$ such that

$$
\begin{aligned}
& B=\left[P^{\prime}\right] B^{\prime} \\
& W_{b^{\prime}}(a)=W_{b}(a)\left[P^{\prime}\right]
\end{aligned}
$$

Generalizing the notion of matrix inverse in the case of finite dimension, $[P]$ and $\left[P^{\prime}\right]$ are inverses in the case of indefinite dimension. More rigorously, $\left[P^{\prime}\right]$ (respectively $[P]$ ) is the left handside inverse (respectively the right handside inverse) of $[P]\left(\right.$ respectively of $\left.\left[P^{\prime}\right]\right)$. They are linked by the relations (see appendix)

$$
\left[P^{\prime}\right][P]=I_{B} \quad[P]\left[P^{\prime}\right]=\mathrm{I}_{B^{\prime}}
$$

in which $I_{B}$ and $I_{B}$, are the $n \times n^{\prime}$ and $n^{\prime} \times n$ identity matrices respectively in the numeral systems with the radices $b$ and $b^{\prime}$. 


\section{Examples: Case of Integer Numbers}

Example 1: let $a$ be the number $(101)_{60}$ in the system of numeration with the radix $b=60$. Let us look for the writing of $a$ in the decimal system (radix $b^{\prime}=10$ ).

The basis change matrix is deduced from the relation

$$
\left[\begin{array}{c}
\cdot \\
60^{3} \\
60^{2} \\
60^{1} \\
60^{0}
\end{array}\right]=\left[\begin{array}{llllll}
\cdot & \cdot & \cdot & \cdot & \cdot & \cdot \\
2 & 1 & 6 & 0 & 0 & 0 \\
0 & 0 & 3 & 6 & 0 & 0 \\
0 & 0 & 0 & 0 & 6 & 0 \\
0 & 0 & 0 & 0 & 0 & 1
\end{array}\right]\left[\begin{array}{c}
10^{5} \\
10^{4} \\
10^{3} \\
10^{2} \\
10^{1} \\
10^{0}
\end{array}\right]
$$

The elements of the basis change matrix which contribute are indicated below

$$
\begin{aligned}
& a=\left[\begin{array}{lllll}
. & 0 & 1 & 0 & 1
\end{array}\right]\left[\begin{array}{l}
60^{3} \\
60^{2} \\
60^{1} \\
60^{0}
\end{array}\right] \\
& =\left[\begin{array}{lllll}
. & 0 & 1 & 0 & 1
\end{array}\right]\left[\begin{array}{cccccc}
\cdot & \cdot & \cdot & . & . & . \\
2 & 1 & 6 & 0 & 0 & 0 \\
0 & 0 & 3 & 6 & 0 & 0 \\
0 & 0 & 0 & 0 & 6 & 0 \\
0 & 0 & 0 & 0 & 0 & 1
\end{array}\right]\left[\begin{array}{c}
0 \\
10^{5} \\
10^{4} \\
10^{3} \\
10^{2} \\
10^{1} \\
10^{0}
\end{array}\right]
\end{aligned}
$$

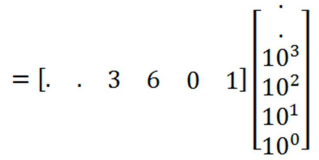

The terms which don't contribute were represented by zeros or dots or blanks when necessary.

It is easy to check

$$
\begin{aligned}
3 \times 10^{3}+6 \times 10^{2}+0 \times 10^{1}+1 \times 10^{0} \\
=1 \times 60^{2}+0 \times 60^{1}+1 \times 60^{0}
\end{aligned}
$$

or

$$
(3601)_{10}=(101)_{60}
$$

We obtain immediately the writing in the numeral system with the radix 10 without remainder because each obtained term is less than $10-1=9$. It will not be the case in the examples 3 and 4.

Example 2: let $a$ be the number (5811) 60 in the numeral system with the radix 60 . Let us look for the writing of $a$ in the decimal system

$$
\begin{aligned}
& a=\left[\begin{array}{lllll}
0 & 0 & 58 & 1 & 1
\end{array}\right]\left[\begin{array}{c}
\dot{\cdot} \\
60^{3} \\
60^{2} \\
60^{1} \\
60^{0}
\end{array}\right] \\
& =\left[\begin{array}{lllll}
0 & 0 & 58 & 1 & 1
\end{array}\right]\left[\begin{array}{cccccc}
2 & 1 & 6 & 0 & 0 & 0 \\
0 & 0 & 3 & 6 & 0 & 0 \\
0 & 0 & 0 & 0 & 6 & 0 \\
0 & 0 & 0 & 0 & 0 & 1
\end{array}\right]\left[\begin{array}{c}
10^{5} \\
10^{4} \\
10^{3} \\
10^{2} \\
10^{0}
\end{array}\right] \\
& =[\ldots(\mathrm{B})(\mathrm{A}) 61]\left[\begin{array}{c}
\vdots \\
10^{5} \\
10^{4} \\
10^{3} \\
10^{2} \\
10^{1} \\
10^{0}
\end{array}\right]
\end{aligned}
$$

The terms designed by (A) and (B)

$$
\begin{aligned}
& (A)=0 \times 0+58 \times 6+1 \times 0+1 \times 0=348 \\
& (B)=0 \times 6+58 \times 3+1 \times 0+1 \times 0=174
\end{aligned}
$$

are greater than $10-1=9$. Then, there are remainders.

By taking the dispositions which respects for (A) and (B) the succession of $b^{i}$ in the basis, we add the elements in the same column in bringing the remainder if necessary to the left handside column which follows immediately (it is the classical rule for addition). Then

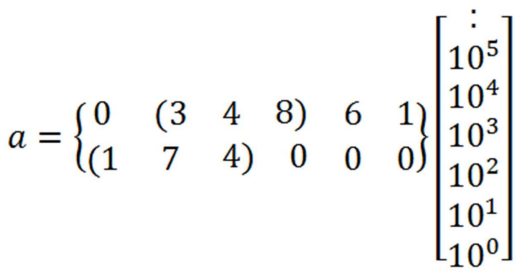

$$
\begin{aligned}
& =\left[\begin{array}{llllll}
2 & 0 & 8 & 8 & 6 & 1
\end{array}\right]\left[\begin{array}{c}
: \\
10^{5} \\
10^{4} \\
10^{3} \\
10^{2} \\
10^{1} \\
10^{0}
\end{array}\right]
\end{aligned}
$$

The answer is 208 861. It is easy to check

$$
\begin{gathered}
60^{2} \times 58+60^{1} \times 1+60^{0} \times 1=2 \times 10^{5}+0 \times 10^{4} \\
+8 \times 10^{3}+8 \times 10^{2}+6 \times 10^{1}+1 \times 10^{0}=208861 \\
(5811)_{60}=(208861)_{10}
\end{gathered}
$$

Example 3: Find the writing of the binary number $(11011)_{2}$ in the decimal system.

The formalism is particularly interesting for the system change concerning the binary system because we have only two figures 0 and 1 .

The basis $B$ and $B^{\prime}$ are writen explicitly for a better undestanding. But in practical calculation, it is sufficient to write the basis change matrix and to take into account the terms which contribute to the calculation

$$
\begin{aligned}
{\left[\begin{array}{lllll}
1 & 1 & 0 & 1 & 1
\end{array}\right]\left[\begin{array}{c}
\vdots \\
2^{4} \\
2^{3} \\
2^{2} \\
2^{1} \\
2^{0}
\end{array}\right] } & =\left[\begin{array}{lllll}
1 & 1 & 0 & 1 & 1
\end{array}\right]\left[\begin{array}{lllll}
0 & 0 & 0 & 1 & 6 \\
0 & 0 & 0 & 0 & 8 \\
0 & 0 & 0 & 0 & 4 \\
0 & 0 & 0 & 0 & 2 \\
0 & 0 & 0 & 0 & 1
\end{array}\right]\left[\begin{array}{c}
10^{4} \\
10^{3} \\
10^{2} \\
10^{1} \\
10^{0}
\end{array}\right] \\
& =\left[\begin{array}{lllll}
0 & 0 & 0 & 1 & (C)
\end{array}\right]\left[\begin{array}{c}
\vdots \\
10^{4} \\
10^{3} \\
10^{2} \\
10^{1} \\
10^{0}
\end{array}\right]
\end{aligned}
$$

The term $(C)=(6 \times 1)+(8 \times 1)+(4 \times 0)+(2 \times$ 1) $+(1 \times 1)=17$ gives 7 in the first column from the right handside with remainder 1 which is to be added to the number 1 of the second column at the left handside to give 
the number 2 .

$$
\begin{aligned}
{\left[\begin{array}{lllll}
1 & 1 & 0 & 1 & 1
\end{array}\right]\left[\begin{array}{c}
\vdots \\
2^{4} \\
2^{3} \\
2^{2} \\
2^{1} \\
2^{0}
\end{array}\right] } & \left.=\left\{\begin{array}{lllll}
0 & 0 & 0 & 1 & (0) \\
0 & 0 & 0 & (1 & 7
\end{array}\right)\right\}\left[\begin{array}{c}
\vdots \\
10^{4} \\
10^{3} \\
10^{2} \\
10^{1} \\
10^{0}
\end{array}\right] \\
& =\left[\begin{array}{lllll}
0 & 0 & 0 & 2 & 7
\end{array}\right]\left[\begin{array}{c}
10^{4} \\
10^{3} \\
10^{2} \\
10^{1} \\
10^{0}
\end{array}\right]
\end{aligned}
$$

It is easy to check

$2 \times 10^{1}+7 \times 10^{0}=1 \times 2^{4}+1 \times 2^{3}+0 \times 2^{2}+1 \times 2^{1}+1 \times 2^{0}$

or

$$
(27)_{10}=(11011)_{2}
$$

Example 4: Find the decimal writing of the octal number $(177606)_{8}$

The basis change matrix is given by the relation

$$
\left[\begin{array}{c}
\vdots \\
8^{5} \\
8^{4} \\
8^{3} \\
8^{2} \\
8^{1} \\
8^{0}
\end{array}\right]=\left[\begin{array}{llllll}
0 & 3 & 2 & 7 & 6 & 8 \\
0 & 0 & 4 & 0 & 9 & 6 \\
0 & 0 & 0 & 5 & 1 & 2 \\
0 & 0 & 0 & 0 & 6 & 4 \\
0 & 0 & 0 & 0 & 0 & 8 \\
0 & 0 & 0 & 0 & 0 & 1
\end{array}\right]\left[\begin{array}{c}
: \\
10^{5} \\
10^{4} \\
10^{3} \\
10^{2} \\
10^{1} \\
10^{0}
\end{array}\right]
$$

The decimal writing of the given octal number can be obtained immediately by performing the matricial product

$$
\begin{aligned}
& {\left[\begin{array}{llllll}
1 & 7 & 7 & 6 & 0 & 6
\end{array}\right]\left[\begin{array}{llllll}
0 & 3 & 2 & 7 & 6 & 8 \\
0 & 0 & 4 & 0 & 9 & 6 \\
0 & 0 & 0 & 5 & 1 & 2 \\
0 & 0 & 0 & 0 & 6 & 4 \\
0 & 0 & 0 & 0 & 0 & 8 \\
0 & 0 & 0 & 0 & 0 & 1
\end{array}\right]}
\end{aligned}
$$

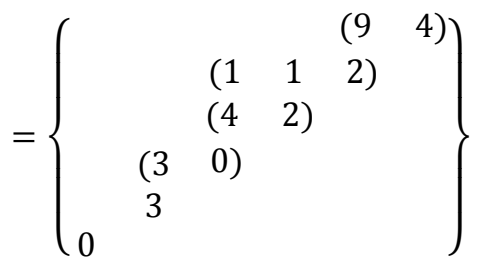

$$
\begin{aligned}
& =\left[\begin{array}{llllll}
0 & 6 & 5 & 4 & 1 & 4
\end{array}\right]
\end{aligned}
$$

The disposition of the figures of the partial sums that we have indicated between brackets should be noted. The figures are added column by column beginning from the right handisde to the left handside and bringing the remainder when needed. It is easy to check that

$$
\begin{aligned}
& 1 \times 8^{5}+7 \times 8^{4}+7 \times 8^{3}+6 \times 8^{2}+0 \times 8^{1}+6 \times 8^{0} \\
& \quad=6 \times 10^{4}+5 \times 10^{3}+4 \times 10^{2}+1 \times 10^{1}+4 \times 10^{0} \\
& \text { Or }(177606)_{8}=(65414)_{10}
\end{aligned}
$$

\section{Generalization}

\subsection{Number with Radix Point But with Finite Numbers After the Radix Point}

We have considered the basis $b^{i}$ with $i \geq 0$ for $\neq 0$, then generalization can be made by taking $i<0$. In the writing of the number, the figures corresponding to $i \geq 0$ and $i<0$ are separated by a point (the radix point)

Example: the number

$$
a=\left[\quad a_{2} \quad a_{1} \quad a_{0} \quad a_{-1} \quad a_{-2} \quad a_{-3}\right]\left[\begin{array}{c} 
\\
b^{2} \\
b^{1} \\
b^{0} \\
b^{-1} \\
b^{-2} \\
b^{-3}
\end{array}\right]
$$

is written in the numeral system with the radix $b$ as the row matrix $\left[\begin{array}{llllll}a_{2} & a_{1} & a_{0} . & a_{-1} & a_{-2} & a_{-3}\end{array}\right]$.

For instance, in the numeral system with the radix $b=2$, the number 101.101 is the row matrix $\left[\begin{array}{llllll}1 & 0 & 1 . & 1 & 0 & 1\end{array}\right]$ representing the expansion

$$
1 \times 2^{2}+0 \times 2^{1}+1 \times 2^{0}+1 \times 2^{-1}+0 \times 2^{-2}+1 \times 2^{-3}
$$

Applying the method described above, we obtain

$$
5 \times 10^{0}+6 \times 10^{-1}+2 \times 10^{-2}+5 \times 10^{-3}
$$

which is written $(5.625)_{10}$ in the decimal system.

Let $s$ be the finite maximum number of the figures not appearing after the point. Multiplying by $b^{s}$, we are taken back to the previous case.

\subsection{Number with Radix Point But with Infinite Number After the Radix Point}

Although the number of figures after the radix point is infinite, the method may again be applied by using approached values by excess and by less: the given number is framed between two finite fixed point numbers. We are taken back to the case 4.1 .

\subsection{Infinite Number at the Left of the Figure Corresponding to $b^{0}$}

The number itself is infinite and has no sense. The method is of course not applicable.

\section{Conclusion}

It is shown in our first paper [1] that the use of matricial formalism can be introduced to deal with the problem of writing numeration and arithmetics. In this second paper, our approach is extended for the study of the problem of numeral system change.

The above results show that our method is very efficient. It gives directly the writing of the number in the new system by a 
more simple and more swift way than the classical method using successive euclidian divisions. The used rules are the current ones of addition, multiplication and matrix calculation (row-column product).

Our method may be applied in the framework of computer science [12].

All along this paper, the basis was represented by a column matrix by decreasing order from the highest values to the lowest ones so that we obtain the current writing of a number as a row matrix, writing from left handside to right handside, following to the ordinary writing by decreasing order (LRd disposition). However, this current writing is inconsistent with adding the remainders from the right handside to the left handside. This inconsistency may be overcome by adopting the writing as row matrix from the left handside to the right handside by increasing order LRi [1]

Appendix: practical calculation

In the paragraph 2 , the relationship between the basis $B$ and $B^{\prime}$ and the basis changes matrices $[P]$ and $\left[P^{\prime}\right]$ was written (relations 7,8 and 9)

$$
\begin{gathered}
B^{\prime}=[P] B \\
B=\left[P^{\prime}\right] B^{\prime} \\
{\left[P^{\prime}\right][P]=\mathrm{I}_{B} a \quad[P]\left[P^{\prime}\right]=\mathrm{I}_{B^{\prime}}}
\end{gathered}
$$

where $\mathrm{I}_{B}$ (respectively $\mathrm{I}_{B^{\prime}}$ ) is unity matrix in the basis $B$ (respectively $B^{\prime}$ ).

We give more details about this remark by expliciting the practical calculation in the case of the example 4 in the paragraph 3. Then the simplicity of the obtention of the results utilizing matricial formalism is shown.

The numeration basis change matrix $[P]$ from the decimal system to the octal system is such that

$$
\left[\begin{array}{c}
\vdots \\
8^{5} \\
8^{4} \\
8^{3} \\
8^{2} \\
8^{1} \\
8^{0} \\
.
\end{array}\right]=[P]\left[\begin{array}{c}
: \\
10^{5} \\
10^{4} \\
10^{3} \\
10^{2} \\
10^{1} \\
10^{0}
\end{array}\right]=\left[\begin{array}{llllll}
0 & 3 & 2 & 7 & 6 & 8 \\
0 & 0 & 4 & 0 & 9 & 6 \\
0 & 0 & 0 & 5 & 1 & 2 \\
0 & 0 & 0 & 0 & 6 & 4 \\
0 & 0 & 0 & 0 & 0 & 8 \\
0 & 0 & 0 & 0 & 0 & 1
\end{array}\right]\left[\begin{array}{c}
: \\
10^{5} \\
10^{4} \\
10^{3} \\
10^{2} \\
10^{1} \\
10^{0} \\
.
\end{array}\right]
$$

Inversely

$$
\left[\begin{array}{c}
: \\
10^{5} \\
10^{4} \\
10^{3} \\
10^{2} \\
10^{1} \\
10^{0}
\end{array}\right]=\left[P^{\prime}\right]\left[\begin{array}{c}
: \\
10^{5} \\
10^{4} \\
10^{3} \\
10^{2} \\
10^{1} \\
10^{0}
\end{array}\right]=\left[\begin{array}{llllll}
3 & 0 & 3 & 2 & 4 & 0 \\
0 & 2 & 3 & 4 & 2 & 0 \\
0 & 0 & 1 & 7 & 5 & 5 \\
0 & 0 & 0 & 1 & 4 & 4 \\
0 & 0 & 0 & 0 & 1 & 2 \\
0 & 0 & 0 & 0 & 0 & 1
\end{array}\right]\left[\begin{array}{c}
\vdots \\
8^{5} \\
8^{4} \\
8^{3} \\
8^{2} \\
8^{1} \\
8^{0}
\end{array}\right]
$$

In practical calculation, we leave void the places occupied by zeros.

Let us perform the products $\left[P^{\prime}\right][P]$ and $[P]\left[P^{\prime}\right]$. For the product $\left[P^{\prime}\right][P]$

$$
\left[P^{\prime}\right][P]=\left[\begin{array}{llllll}
3 & 0 & 3 & 2 & 4 & 0 \\
0 & 2 & 3 & 4 & 2 & 0 \\
0 & 0 & 1 & 7 & 5 & 5 \\
0 & 0 & 0 & 1 & 4 & 4 \\
0 & 0 & 0 & 0 & 1 & 2 \\
0 & 0 & 0 & 0 & 0 & 1
\end{array}\right]\left[\begin{array}{llllll}
0 & 3 & 2 & 7 & 6 & 8 \\
0 & 0 & 4 & 0 & 9 & 6 \\
0 & 0 & 0 & 5 & 1 & 2 \\
0 & 0 & 0 & 0 & 6 & 4 \\
0 & 0 & 0 & 0 & 0 & 8 \\
0 & 0 & 0 & 0 & 0 & 1
\end{array}\right]
$$

By performing the calculation in the decimal basis, we find

$$
\left[P^{\prime}\right][P]=\left[\begin{array}{llllll}
1 & 0 & 0 & 0 & 0 & 0 \\
0 & 1 & 0 & 0 & 0 & 0 \\
0 & 0 & 1 & 0 & 0 & 0 \\
0 & 0 & 0 & 1 & 0 & 0 \\
0 & 0 & 0 & 0 & 1 & 0 \\
0 & 0 & 0 & 0 & 0 & 1
\end{array}\right]=I_{B}
$$

where $I_{B}$ is obviously the identity matrix in the decimal system.

For the calculation of the product $[P]\left[P^{\prime}\right]$, there are two possible methods:

i) We may perform the matrix product in decimal basis and after convert the result in the octal system.

ii) We may convert the elements of the matrices in octal system and then perform the matrix product in the octal system (using octal multiplication and addition tables: see below.)

The two possible methods lead to the same result $\left[P^{\prime}\right][P]=I_{B^{\prime}}$ with $I_{B^{\prime}}$ the identity matrix in the octal system. If the calculation is performed in the octal system, we need the octal system multiplication and addition tables.

For octal system multiplication table

\begin{tabular}{|l|l|l|l|l|l|l|l|l|}
\hline$\times$ & 0 & 1 & 2 & 3 & 4 & 5 & 6 & 7 \\
\hline 0 & 0 & 0 & 0 & 0 & 0 & 0 & 0 & 0 \\
\hline 1 & 0 & 1 & 2 & 3 & 4 & 5 & 6 & 7 \\
\hline 2 & 0 & 2 & 4 & 6 & 10 & 12 & 14 & 16 \\
\hline 3 & 0 & 3 & 6 & 11 & 14 & 17 & 22 & 25 \\
\hline 4 & 0 & 4 & 10 & 14 & 20 & 24 & 30 & 34 \\
\hline 5 & 0 & 5 & 12 & 17 & 24 & 31 & 36 & 43 \\
\hline 6 & 0 & 6 & 14 & 22 & 30 & 36 & 44 & 52 \\
\hline 7 & 0 & 7 & 16 & 25 & 34 & 43 & 52 & 61 \\
\hline
\end{tabular}

For the octal system addition table

\begin{tabular}{|l|l|l|l|l|l|l|l|l|}
\hline+ & 0 & 1 & 2 & 3 & 4 & 5 & 6 & 7 \\
\hline 0 & 0 & 1 & 2 & 3 & 4 & 5 & 6 & 7 \\
\hline 1 & 1 & 1 & 3 & 4 & 5 & 6 & 7 & 10 \\
\hline 2 & 2 & 3 & 4 & 5 & 6 & 7 & 10 & 11 \\
\hline 3 & 3 & 4 & 5 & 6 & 7 & 10 & 11 & 12 \\
\hline 4 & 4 & 5 & 6 & 7 & 10 & 11 & 12 & 13 \\
\hline 5 & 5 & 6 & 7 & 10 & 11 & 12 & 13 & 14 \\
\hline 6 & 6 & 7 & 10 & 11 & 12 & 13 & 14 & 15 \\
\hline 7 & 7 & 10 & 11 & 12 & 13 & 14 & 15 & 16 \\
\hline
\end{tabular}

The results in the example 4 can be verified as well. We can show that the number $(177606)_{8}$ in octal system is written $(65414)_{10}$ in decimal system and reciprocally. The basis change matrices are respectively $[P]$ and $\left[P^{\prime}\right]$.

$$
\left[\begin{array}{llllll}
1 & 7 & 7 & 6 & 0 & 6
\end{array}\right][P]=\left[\begin{array}{llllll}
0 & 6 & 5 & 4 & 1 & 4
\end{array}\right]
$$

All operations are, of course, performed in the decimal system. Inversely, we can also find after performing 
calculation in the octal system that

$$
\left[\begin{array}{llllll}
0 & 6 & 5 & 4 & 1 & 4
\end{array}\right]\left[P^{\prime}\right]=\left[\begin{array}{llllll}
1 & 7 & 7 & 6 & 0 & 6
\end{array}\right]
$$

\section{References}

[1] Raoelina Andriambololona, Ravo Tokiniaina Ranaivoson, Wilfrid Chrysante Solofoarisina. "Arithmetics and Matricial Calculation", Science Publishing Group, Pure and Applied Mathematics Journal (on press), 2016.

[2] Raoelina Andriambololona, "Algèbre linéaire et multilinéaire", Collection LIRA, INSTN-Madagascar, Antananarivo, Madagascar, 1986.

[3] Anton Howard, Chris Rorres, "Elementary Linear Algebra" (10th ed.), John Wiley \& Sons, 2010.

[4] William C. Brown "Matrices and vector spaces", New York, NY: Marcel Dekker, 1991.

[5] Georges Ifrah, David Bellos, E. F. Harding, Sophie Wood, Ian Monk, "The Universal History of Numbers: From Prehistory to the Invention of the Computer", John Wiley \& Sons, New York, 1999.

[6] Stephen Chrisomalis, "Numerical Notation: A Comparative History”, Cambridge University Press, 2010.
[7] Anton Glaser, "History of binary and other nondecimal numeration", Tomash, 1971.

[8] M. Morris Mano, Charles Kime. "Logic and computer design fundamentals." (4th ed.). Pearson, 2014.

[9] Raoelina Andriambololona, "Théorie générale des numérations écrite et parlée". Bull. Acad. Malg. LXIV./1-2, Antananarivo, Madagascar, 1986.

[10] Raoelina Andriambololona, "Théorie générale des numérations écrite et parlée. II Utilisation du calcul matriciel en arithmétique. Nouvelle proposition d'écriture, d'énoncé des règles d'addition et de multiplication des nombres.". Bull. Acad. Malg LXV/1-2, Antananarivo, Madagascar, 1987.

[11] Raoelina Andriambololona, "Théorie générale des numérations écrite et parlée. II- Utilisation du calcul matriciel en arithmétique. Application au changement de bases de numération. Bull. Acad. Malg. LXV./1-2, Antananarivo, Madagascar”, 1987 (1989).

[12] Raoelina Andriambololona, Hanitriarivo Rakotoson "Mpikajy elekronika sy siantifika mampiasa ny fomba fanisana Malagasy (Electronic and scientific calculator based on malagasy counting method)", communication at the Academie Malgache, Antananarivo Madagascar, 05 June 2008. 